\title{
Population Structure and Antimicrobial Resistance of Invasive Serotype IV Group B Streptococcus, Toronto, Ontario, Canada
}

\author{
Sarah Teatero, Allison McGeer, Aimin Li, Janice Gomes, Christine Seah, Walter Demczuk, \\ Irene Martin, Jessica Wasserscheid, Ken Dewar, Roberto G. Melano, Nahuel Fittipaldi
}

\begin{abstract}
We recently showed that $37 / 600(6.2 \%)$ invasive infections with group B Streptococcus (GBS) in Toronto, Ontario, Canada, were caused by serotype IV strains. We report a relatively high level of genetic diversity in 37 invasive strains of this emerging GBS serotype. Multilocus sequence typing identified 6 sequence types (STs) that belonged to 3 clonal complexes. Most isolates were ST-459 (19/37, 51\%) and ST-452 (11/37, 30\%), but we also identified ST-291, ST-3, ST-196, and a novel ST-682. We detected further diversity by performing whole-genome single-nucleotide polymorphism analysis and found evidence of recombination events contributing to variation in some serotype IV GBS strains. We also evaluated antimicrobial drug resistance and found that ST-459 strains were resistant to clindamycin and erythromycin, whereas strains of other STs were, for the most part, susceptible to these antimicrobial drugs.
\end{abstract}

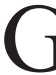
roup B Streptococcus (GBS), also known as StreptoJ coccus agalactiae, are a major cause of neonatal sepsis and meningitis and are increasingly being associated with invasive infections in nonpregnant adults (1-3). For instance, in 2011, adult cases accounted for nearly $90 \%$ of the burden of GBS disease in the United States (4). Elderly persons and those with preexisting conditions (e.g., diabetes mellitus, cirrhosis, cancer, and compromised immunity) are most at risk (5). The clinical features of GBS disease in adults range from localized tissue infection to

Author affiliations: Public Health Ontario, Toronto, Ontario, Canada (S. Teatero, A. Li, J. Gomes, C. Seah, R.G. Melano, N. Fittipaldi); University of Toronto, Toronto (A. McGeer, R.G. Melano, N. Fittipaldi); Mount Sinai Hospital, Toronto (A. McGeer, R.G. Melano); Public Health Agency of Canada, Winnipeg, Manitoba, Canada (W. Demczuk, I. Martin); McGill University, Montreal, Quebec, Canada (J. Wasserscheid, K. Dewar); Genome Quebec Innovation Centre, Montreal (J. Wasserscheid, K. Dewar)

DOI: http://dx.doi.org/10.3201/eid2104.140759 severe bacteremia with shock (1). Less common clinical syndromes, such as endocarditis and meningitis, are associated with considerable illness and death (1).

GBS are classified into 10 serotypes (Ia, Ib, and IIIX) on the basis of a serologic reaction against capsular polysaccharide. The most common GBS serotypes causing invasive disease in the United States and Canada in adults and neonates are Ia, III, and V (2,3,6-9). However, recent reports have shown that serotype IV GBS is emerging in pregnant carriers and causing infections in neonates and adults in North America and several other regions (10-14). This emergence is of concern because GBS conjugate vaccines that are being developed to prevent invasive disease may protect only against serotypes $\mathrm{Ia}, \mathrm{Ib}, \mathrm{II}, \mathrm{III}$, and $\mathrm{V}$, or combinations thereof $(15,16)$.

Ferrieri et al. reported different genetic backgrounds among serotype IV GBS strains, which have been shown to include sequence types (STs) ST-452 and ST-459 (11). Horizontal gene transfer occurs frequently in GBS. For example, capsular switching from serotype III to IV has been recently reported and found to be the result of recombination involving the entire cps locus $(8,17,18)$. GBS are considered to be universally susceptible to penicillins; therefore, these are the primary drugs used for prophylaxis and treatment of GBS disease $(19,20)$. However, macrolide and lincosamide antimicrobial drugs, such as erythromycin and clindamycin, are often used to treat GBS infections in patients allergic or suspected to be allergic to $\beta$-lactam drugs. Acquisition of resistance against these drugs by horizontal gene transfer has been frequently described in $\operatorname{GBS}(2,21)$.

We recently reported a relatively high prevalence $(6.2 \%)$ of serotype IV strains among GBS causing invasive infections in Toronto, Ontario, Canada (8). In the current study, we used whole-genome sequencing (WGS) to characterize the population structure of serotype IV GBS isolates. We describe a genetically heterogeneous population of isolates that differ in their antimicrobial drug resistance profiles. 


\section{Methods}

\section{Bacterial Strains, Culture Conditions,} DNA Preparations, Serotyping, and Pilus Typing

We used 37 serotype IV GBS strains collected during 2009-2012 by the Toronto Invasive Bacterial Disease Network, a population-based surveillance system for invasive bacterial diseases in metropolitan Toronto and the Peel region in Ontario, Canada (total population under surveillance $\approx 5.5$ million persons in 2011). Laboratorybased surveillance involves the 28 hospitals providing care to and the 25 laboratories processing sterile site cultures for residents of the population area; laboratory personnel submit all GBS isolates from sterile sites to the central study laboratory. The sample was composed of all available serotype IV GBS strains determined by latex agglutination and corresponded to $6.2 \%$ of GBS identified during the study by the program (8). Only 1 strain per patient was included in the collection; there was no clear evidence of localized outbreaks caused by serotype IV GBS during the collection period. Strains are listed in online Technical Appendix Table 1 (http://wwwnc.cdc. gov/EID/article/21/4/14-0759-Techapp1.pdf).

Bacteria were grown at $37^{\circ} \mathrm{C}$ in an atmosphere of $5 \%$ $\mathrm{CO}_{2}$ on Columbia agar plates supplemented with 5\% sheep blood. Genomic DNA was obtained by using the QIAamp DNA Minikit (QIAGEN, Toronto, Ontario, Canada) according to the manufacturer's protocol for gram-positive organisms. The presence of pilus islands PI-1, PI-2a, and $\mathrm{PI}-2 \mathrm{~b}$ was determined by using PCR as described (22).

\section{WGS, Bioinformatics Methods, and Phylogenetic Analysis}

WGS libraries were prepared for all 37 isolates by using Nextera XT Kits (Illumina, San Diego, CA, USA) and sequenced as paired-end reads with either Illumina HiSeq 2500 (100 bp $+100 \mathrm{bp})$ or MiSeq (150 bp $+150 \mathrm{bp})$ instruments (data are available at the Sequence Read Archive under accession no. SRP040805). Parsing of the multiplexed sequencing reads and removal of barcode information was performed by using onboard software. STs were derived directly from short reads by using SRST2 (https://github. $\mathrm{com} / \mathrm{katholt} / \mathrm{srst} 2)$. Ambiguous or novel alleles were confirmed by PCR and Sanger sequencing.

Neighbor-joining phylogenetic trees $(1,000$ bootstrap replications) were then generated by using SplitsTree4 (23) and the concatenated sequences of the 7 loci used in the GBS multilocus sequence typing (MLST) scheme. For WGSbased phylogenies, we sequenced to closure the genomes of ST-459 strain NGBS061 and ST-452 strain NGBS572 (GenBank accession nos. CP007631 and CP007632, respectively) by using single-molecule real-time (SMRT) sequencing (Pacific Biosciences, Menlo Park, CA, USA).
Two SMRT cells of sequence were generated for each isolate, which generated $>220 \mathrm{Mb}$ of data in reads $>3 \mathrm{~kb}$ (37,218 reads, average length $6.1 \mathrm{~kb}$ for NGBS061; 54,803 reads, average length $5.6 \mathrm{~kb}$ for NGBS572). In brief, we used HGAP version 2 (24) to correct the long reads and Celera Assembler 7.0 (25) to assemble the corrected reads, followed by 2 rounds of polishing with Quiver (https:// github.com/PacificBiosciences/GenomicConsensus). Coverage of final assemblies in reads $>3 \mathrm{~kb}$ was $84 \times$ for NGBS061 and 111× for NGBS572.

To assess base-calling accuracy in the Pacific Biosciences assembly, Illumina short reads for the 2 isolates were aligned to their respective assemblies by using BLAT (26). Both genome assemblies were completely concordant with full-length perfectly aligning Illumina short-reads, except for NGBS061 in a region of increased AT richness, which had reduced Illumina coverage. For consistency, both genome assemblies were formatted to begin at the first nucleotide of the intergenic region immediately preceding the gene encoding the chromosomal replication initiation protein (dnaA; Genbank accession no. YP-328728).

Illumina short reads of all other isolates were aligned to the reference genomes, and coverage relative to the reference genome was calculated by using Mosaik (https:// code.google.com/p/mosaik-aligner/). Polymorphisms were identified by using VAAL (27). A matrix file containing genotypes of all strains at each polymorphic locus was created from the VAAL polymorphism output data by using a custom script. Then, for each individual strain, singlenucleotide polymorphisms (SNPs) were concatenated in order of occurrence relative to the genome of the corresponding reference strain (see Results). Neighbor-joining phylogenetic trees ( 1,000 bootstrap replications) were then generated with SplitsTree4.

The A5 pipeline was used for de novo assembly of Illumina-sequenced GBS strains (28). Areas of recombination of the ST-196 strains NGBS447 and NGBS472 were defined by using BratNextGen software (29) run with 20 iterations and 100 replicates, using a p value of 0.05 as the significance cutoff. Genome visualizations were created by using BRIG (30) and edited by using Adobe Illustrator (Adobe Systems Inc., San Jose, CA, USA).

\section{Antimicrobial Drug Susceptibility}

MICs for penicillin, ampicillin, cefotaxime, levofloxacin, tetracycline, erythromycin, and clindamycin were determined by using the agar dilution method according to the Clinical and Laboratory Standards Institute (31). We also used SRST2 and a database of 1,913 variants of genes encoding antimicrobial drug resistance (https://github.com/ katholt/srst2) to test for genetic determinants of antimicrobial drug resistance in genomes of serotype IV strains. 


\section{Results}

\section{Isolate Source and Age Distribution of Patients}

The 37 serotype IV GBS isolates used in this study represent $6.2 \%$ of 600 GBS strains isolated in the Toronto-Peel metropolitan area of Ontario during 2009-2012 (8). One isolate was collected in 2009, 15 isolates in 2010, 9 isolates in 2011, and 12 isolates in 2012. Most isolates were from invasive cases in adults (19-59 years of age, $n=14$ ) or older adults $(\geq 60$ years of age, $n=21)$ patients. We did not observe serotype IV GBS in neonates with early-onset disease $(<7$ days of age). However, 1 isolate was obtained from a child with late-onset disease (7-89 days of age), and an additional strain was isolated from a child 188 days of age. Most (34/37) serotype IV isolates were collected from blood; only 1 isolate was collected from soft tissue, and 2 isolates were collected from synovial fluid.

\section{Genetic Diversity of Serotype IV GBS Strains}

To assess genetic diversity of serotype IV GBS strains in our collection, we sequenced the genomes of all 37 strains and used SRST2 to derive MLST information directly from the short-read WGS data. We identified 6 STs representing 3 clonal complexes (CCs), which is indicative of a relatively high level of genetic diversity among our isolates. Two isolates were ST-291 included in hypervirulent CC17; 11 isolates were ST-452 (CC23); 2 isolates were ST-3 (CC1); and 1 isolate was a novel ST-682 included in CC1. Most (19/37) strains belonged to ST-459 (CC1). We also identified 2 strains of ST-196, a closely related single locus variant ( $s d h A$ gene) of ST-459. The 2 STs comprising most strains (ST-452 and ST-459) were also the most genetically distant, as shown by neighbor-joining phylogenetic analysis of concatenated sequences of alleles at all MLST loci (Figure 1, panel A).

To further understand genetic differences between these 2 STs, we arbitrarily chose 1 ST-452 strain (NGBS572) and 1 ST-459 strain (NGBS061) and sequenced their genomes to closure by using Pacific Biosciences SMRT technology. The genomes were circular chromosomes of 2,061,426 bp and 2,221,207 bp, respectively (online Technical Appendix Figure 1). We did not find evidence of any associated plasmids in either isolate. However, we used an SMRT sequencing protocol with a DNA size-selection step for DNA fragments $\geq 20 \mathrm{~kb}$, which might have removed small plasmids that could have been present. The difference in genome size between the strains was explained primarily by the smaller number and size of mobile genetic elements found in NGBS572 than in NGBS061 (online Technical Appendix Figure 1). The core genomes of NGBS572 and NGBS061 (i.e., $\approx 1$.9-Mbp portion of the genome lacking mobile genetic elements that is conserved in gene content between both strains) differed by 13,158 biallelic SNPs and 852 insertions or deletions (indels).

We next investigated genetic diversity within the ST452 and ST-459 groups. Both groups were relatively homogeneous. On average, ST-452 strains differed from the ST-452 reference strain NGBS572 by only 45 SNPs and 10 indels in the core genome. On average, ST-459 strains had only 68 SNPs and 16 indels relative to the core genome of the ST-459 reference strain NGBS061. Neighbor-joining phylogenetic trees constructed by using concatenated SNPs in the core genome showed additional but limited diversity among isolates within each ST and some clustering within ST-459 (Figure 1, panels B,C).

Other STs in our collection had either 1 or 2 strains. Thus, we chose not to sequence these genomes to closure to assess genetic diversity but instead identified polymorphisms relative to the ST-452 and ST-459 reference strains. Both ST-3 strains showed a comparable number of polymorphisms against each reference strain (online Technical Appendix Table 2). Similar results were observed for each of the ST-291 strains (i.e., both strains differed from the ST452 and ST-459 reference strains by a comparable number of polymorphisms) (online Technical Table 2). In contrast, the 2 ST-196 strains greatly differed between themselves in the number of polymorphisms separating them from the reference strains (online Technical Appendix Table 2). One ST-196 strain, strain NGBS447, had 384 core SNPs and 60 core indels relative to the ST-459 reference strain, where the second ST-196 strain, NGBS472, had 1689 core SNPs and 124 core indels relative to the ST-459 reference strain. Further analysis discovered that the excess polymorphisms in NGBS472 were not evenly distributed across the genome. Instead, some discrete regions of the genome showed an overabundance of polymorphisms, which is suggestive of recombination.

Using BratNextGen, we precisely defined these regions of recombination (online Technical Appendix Figure 2). When these regions of recombination were excluded from the analysis, strain NGBS447 had 107 SNPs and 16 indels relative to the ST-459 reference strain and NGBS472 had 281 SNPs and 128 indels relative to the same reference genome. Genome regions having undergone recombination in strain NGBS472 included genes encoding major virulence factors, such as the 2-component system $\operatorname{csrRS}$ (32) and pili (33). Pili in GBS are encoded by genes found in 2 distinct genome locations and organized in so-called pilus island 1 (PI-1) and PI-2. PI-2 has 2 variants (PI-2a or PI-2b) (33). One of the ST-196 strains had a pilus profile of PI-1 + PI-2a, and the other had a profile of PI-1 + PI-2b (online Technical Appendix Figure 3). Except for these ST-196 strains, strains of the different STs had consistent pilus island profiles (online Technical Appendix Figure 3). 


\section{Antimicrobial Drug Susceptibility of Serotype IV GBS Strains}

All serotype IV GBS strains were susceptible to all $\beta$-lactams, vancomycin, and levofloxacin. We observed resistance to erythromycin and clindamycin in $20 / 37$ $(54 \%)$ and $19 / 37(51 \%)$ of the isolates, respectively; 23 $(62 \%)$ of the isolates were resistant to tetracycline. The proportion of resistance and susceptibility, as well as $50 \%$ MICs and 90\% MICs, are shown in online Technical Appendix Table 3. All ST-459 isolates were resistant to clindamycin and erythromycin; ST-452 isolates were susceptible to these antimicrobial drugs (Figure 2; online Technical Appendix Table 4). Strains of other STs were susceptible to these 2 drugs, except 1 of the ST-291 strains, which was resistant to erythromycin but susceptible to clindamycin (Figure 2; online Technical Appendix Table 4). Macrolide resistance in ST-459 strains is probably attributable to the action of ermTR, a gene that was detected in all ST-459 strains (online Technical Appendix Table 1). We also identified the ermT gene in the genome of the erythromycin-resistant ST-291 strain (online Technical Appendix Table 1). Resistance to tetracycline correlated with presence of the tet $M$ gene (online Technical Appendix Table 1) and was observed among strains of all STs except ST-452. However, we identified 3 ST-459 strains that were susceptible to tetracycline (Figure 2; online Technical Appendix Table 4). One of these had the tet $M$ gene, but genome data showed a 7-nt deletion in the gene, which was predicted to result in early termination of translation. We did not detect tet genes in the other 2 ST459 strains (online Technical Appendix Table 1).

\section{Discussion}

The prevalence of serotype IV GBS is increasing globally among pregnant women; infections in neonates and invasive infections in adults are also increasing. Large proportions of serotype IV GBS isolates have been reported among carrier isolates in the United Arab Emirates (26.3\%) and Turkey (8.3\%) (34,35). In Zimbabwe, serotype IV strains accounted for $5.1 \%$ of GBS isolates from carrier pregnant women and $4.0 \%$ of all GBS strains isolated from hospitalized patients (36). A recent report found a relatively high percentage (16\%) of serotype IV strains among cases of GBS early-onset disease in Minnesota (11). In a longitudinal study in the United States, the proportion of this serotype among all GBS strains isolated from nonpregnant adults increased from $0.2 \%$ in $1998-1999$ to $5.7 \%$ in 2005-2006 (3).

In Canada, a country-wide survey conducted in 1996 found no serotype IV GBS cases in 79 nonpregnant adult women (9). However, we recently reported that serotype IV GBS in the greater Toronto area during 2009-2012 accounted for $6.2 \%$ of all invasive GBS infections (8). Cases

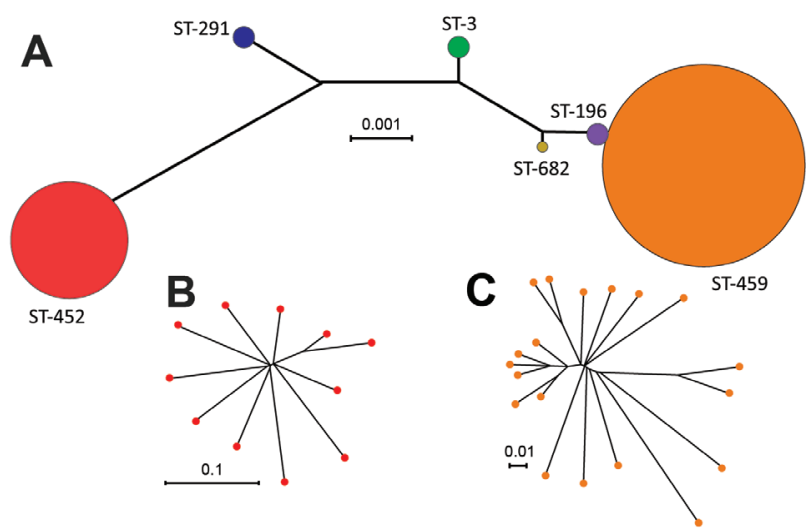

Figure 1. Inferred genetic relationships of invasive serotype IV group B Streptococcus (GBS), Toronto, Ontario, Canada. A) Neighbor-joining phylogenetic tree constructed by using the concatenated sequences of the 7 gene loci ( $s d h A$, adhP, tkt, glcK, atr, pheS, and $g \ln A$ ) used in the multilocus sequence typing (MLST) scheme for GBS. Each circle represents a single MLST sequence type (ST); circle colors differentiate the 6 STs found among strains in our collection and their sizes are proportional to the number of isolates. B) Neighbor-joining phylogenetic analysis based on concatenated sequences of 316 nonredundant singlenucleotide polymorphism (SNP) loci identified by whole-genome sequencing relative to the core genome ST-452 reference strain NGBS572, which shows further diversity within ST-452. C) Diversity within ST-459 serotype IV GBS shown by neighborjoining phylogenetic analysis based on concatenated sequences of 526 nonredundant SNP loci relative to the core genome of ST-459 reference strain NGBS061. Scale bars indicate nucleotide substitutions per site.

in children were rare, and most (94\%) serotype IV GBS invasive infections in Toronto were in adults $\geq 19$ years of age. It may be possible that the low incidence of serotype IV in neonates, and the absence of cases of early-onset disease, is a reflection of a low prevalence of colonizing serotype IV GBS in women of childbearing age. Future studies to determine the prevalence of serotype IV GBS in pregnant women will be useful in elucidating the reasons for this apparent absence of serotype IV GBS neonatal invasive disease.

Given the restricted geographic and temporal origin of our collection of invasive serotype IV GBS strains, we hypothesized that their emergence might represent the successful expansion of a single clone. However, other GBS serotypes usually encompass strains with different genetic backgrounds that might have different tissue tropism and virulence potential (37). Thus, an alternative hypothesis was that our serotype IV GBS collection was composed of strains of diverse genetic backgrounds and that $\geq 1$ clones underwent expansion.

To begin to differentiate between these and other hypotheses, we conducted WGS and evaluated the population structure of our collection. Results showed a relatively high level of genetic diversity among the 37 serotype IV 


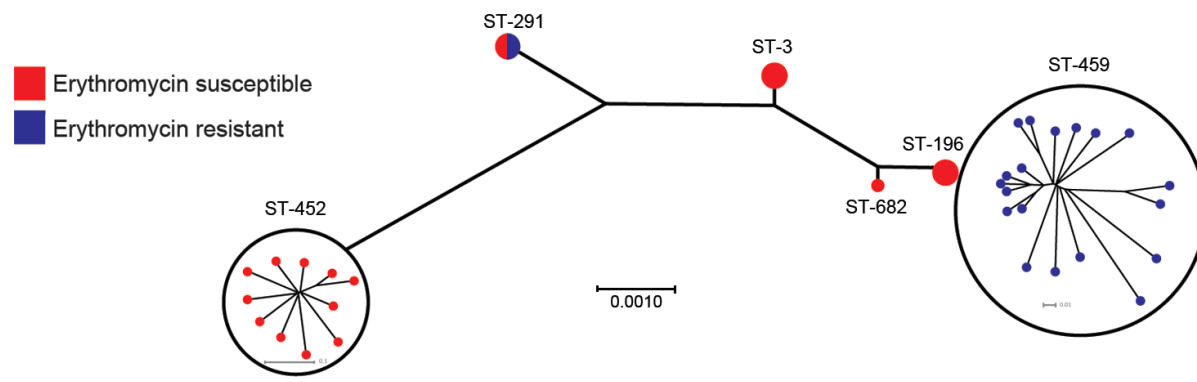

Figure 2. Susceptibility to erythromycin, clindamycin, and tetracycline among serotype IV group B Streptococcus and sequence types (STs), Toronto, Ontario, Canada. All ST-459 strains and 1 ST-291 strain were resistant to erythromycin; we detected ermTR and ermT genes in all erythromycinresistant strains (online Technical Appendix, http://wwwnc.cdc. gov/EID/article/21/4/14-0759Techapp1.pdf). Resistance to clindamycin was observed only among ST-459 strains. Resistance to tetracycline was common among all STs except ST-452 and correlated with presence of the tetM gene in isolates (online Technical Appendix). Scale bars indicate nucleotide substitutions per site.

Tetracycline susceptible

Tetracycline resistant

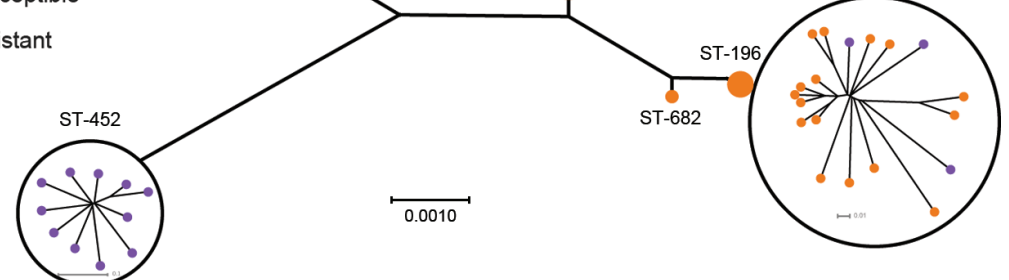

GBS isolates, which were assigned to 6 MLST STs and 3 CCs. Two of our invasive serotype IV isolates belonged to ST-291, a member of the hypervirulent CC17. Previous studies have shown that several strains of this ST have acquired a serotype IV capsule by recombination involving exchange of the full capsule-encoding cps locus $(8,17)$. We also found 2 ST-3 strains and a novel ST-682 strain, both included in $\mathrm{CC}$, one of the major CCs associated with human GBS disease. Our collection contained 2 strains of ST-196 (CC1). WGS showed that the genomes of these 2 ST-196 strains differed extensively, and further analysis indicated that these genome differences were likely caused by recombination. These findings highlight the major role of horizontal gene exchange of extensive regions of DNA in GBS diversification (38) and expose the limitations of MLST to fully identify GBS diversity.

ST-452 (CC23) and ST-459 (CC1) were the 2 most common STs in our collection. These 2 STs have recently been described and were found in invasive serotype IV strains that caused disease in Minnesota $(10,11)$. The reasons why these 2 highly divergent genomic backgrounds seemingly thrive among serotype IV GBS strains that caused invasive disease in Toronto remain to be investigated. However, SNP analysis showed a low level of genetic diversity among strains of each ST-452 and ST-459, which is compatible with recent emergence and ongoing clonal expansion. These STs were also the most phylogenetically distant groups in our collection. We showed that ST-452 and ST-459 genomes differ greatly in size, largely because they have different mobile genetic elements, such as prophages and different pilus islands. The core genomes of these 2 most prevalent STs also differed considerably, as shown by SNP analysis.

Another difference among the groups was that ST-459 strains were resistant to clindamycin and erythromycin, and for the most part to tetracycline, whereas ST-452 isolates were susceptible to these drugs. We associated resistance to macrolides with presence of the ermTR gene in 
all ST-459 strains. The increase in the number of cases of invasive disease caused by serotype IV isolates and concurrent emergence of clindamycin resistance among a successful clone of this serotype could be an early indication of the beginning of clinical problems similar to those observed for serotype V GBS (39). Resistance to erythromycin was observed in 1 ST-291 strain and correlated with presence of the ermT gene. Tetracycline resistance was observed in $23(62 \%)$ of the serotype IV GBS isolates, and in most instances correlated with presence of the tet $M$ gene.

Capsular switching has been described frequently in GBS $(22,40)$. At least in part, our results suggest that different lineages of serotype IV GBS have independently acquired a serotype IV capsule from unidentified donors by recombination. As the global presence of serotype IV continues to increase, monitoring of this emerging serotype is warranted. Conjugate vaccines that are being developed should include polysaccharide and/or associated GBS virulence proteins of this serotype, or they risk not being completely protective against the full range of GBS disease.

\section{Acknowledgments}

We thank Taryn B.T. Athey for assistance with bioinformatics analysis and genome annotations and Dax Torti for performing Illumina sequencing of our strains. During this study, we used the S. agalactiae MLST website (http://pubmist.org/ sagalactiae/), which was developed by Keith Jolley and is sited at the University of Oxford.

This study was supported by Public Health Ontario. The MLST website is supported by the Wellcome Trust.

Ms. Teatero is a research technician at Public Health Ontario, Toronto, Ontario, Canada. Her primary research interest is the molecular epidemiology of GBS.

\section{References}

1. Farley MM. Group B streptococcal disease in nonpregnant adults. Clin Infect Dis. 2001;33:556-61. http://dx.doi.org/10.1086/322696

2. Phares CR, Lynfield R, Farley MM, Mohle-Boetani J, Harrison LH, Petit S, et al. Epidemiology of invasive group B streptococcal disease in the United States, 1999-2005. JAMA. 2008;299:205665. http://dx.doi.org/10.1001/jama.299.17.2056

3. Skoff TH, Farley MM, Petit S, Craig AS, Schaffner W, Gershman K, et al. Increasing burden of invasive group B streptococcal disease in nonpregnant adults, 1990-2007. Clin Infect Dis. 2009;49:85-92. http://dx.doi.org/10.1086/599369

4. Centers for Disease Control and Prevention. Active bacterial core surveillance report, Emerging Infections Program Network, group B Streptococcus, 2011. 2012 [cited 2014 Mar 26]. http://www.cdc.gov/abcs/reports-findings/survreports/gbs11.pdf

5. Jackson LA, Hilsdon R, Farley MM, Harrison LH, Reingold AL, Plikaytis BD, et al. Risk factors for group B streptococcal disease in adults. Ann Intern Med. 1995;123:415-20. http://dx.doi.org/10.7326/0003-4819-123-6-199509150-00003

6. Davies HD, Raj S, Adair C, Robinson J, McGeer A, Alberta GBSSG. Population-based active surveillance for neonatal group
B streptococcal infections in Alberta, Canada: implications for vaccine formulation. Pediatr Infect Dis J. 2001;20:879-84. http://dx.doi.org/10.1097/00006454-200109000-00011

7. Kothari NJ, Morin CA, Glennen A, Jackson D, Harper J, Schrag SJ, et al. Invasive group B streptococcal disease in the elderly, Minnesota, USA, 2003-2007. Emerg Infect Dis. 2009;15:1279-81. http://dx.doi.org/10.3201/eid1508.081381

8. Teatero S, McGeer A, Low DE, Li A, Demczuk W, Martin I, et al. Characterization of invasive group B Streptococcus from the greater Toronto area, Canada. J Clin Microbiol. 2014. 52:1441-7. http://dx.doi.org/10.1128/JCM.03554-13

9. Tyrrell GJ, Senzilet LD, Spika JS, Kertesz DA, Alagaratnam M, Lovgren M, et al. Invasive disease due to group B streptococcal infection in adults: results from a Canadian, population-based, active laboratory surveillance study-1996. Sentinel Health Unit Surveillance System Site Coordinators. J Infect Dis. 2000; 182:168-73. http://dx.doi.org/10.1086/315699

10. Diedrick MJ, Flores AE, Hillier SL, Creti R, Ferrieri P. Clonal analysis of colonizing group B Streptococcus, serotype IV, an emerging pathogen in the United States. J Clin Microbiol. 2010;48:3100-4. http://dx.doi.org/10.1128/JCM.00277-10

11. Ferrieri P, Lynfield R, Creti R, Flores AE. Serotype IV and invasive group B Streptococcus disease in neonates, Minnesota, USA, 2000-2010. Emerg Infect Dis. 2013;19:551-8.

12. Jannati E, Roshani M, Arzanlou M, Habibzadeh S, Rahimi G, Shapuri R. Capsular serotype and antibiotic resistance of group B streptococci isolated from pregnant women in Ardabil, Iran. Iran J Microbiol. 2012;4:130-5.

13. Kiely RA, Cotter L, Mollaghan AM, Cryan B, Coffey A, Lucey B. Emergence of group B Streptococcus serotype IV in women of child-bearing age in Ireland. Epidemiol Infect. 2011;139:236-8. http://dx.doi.org/10.1017/S0950268810001275

14. Palmeiro JK, Dalla-Costa LM, Fracalanzza SE, Botelho AC, da Silva Nogueira K, Scheffer MC, et al. Phenotypic and genotypic characterization of group B streptococcal isolates in southern Brazil. J Clin Microbiol. 2010;48:4397-403. http://dx.doi. org/10.1128/JCM.00419-10

15. Madhi SA, Dangor Z, Heath PT, Schrag S, Izu A, Sobanjo-Ter Meulen A, et al. Considerations for a phase-III trial to evaluate a group B Streptococcus polysaccharide-protein conjugate vaccine in pregnant women for the prevention of early- and lateonset invasive disease in young-infants. Vaccine. 2013;31(Suppl 4):D52-7. http://dx.doi.org/10.1016/j.vaccine.2013.02.029

16. Paoletti LC, Kasper DL. Glycoconjugate vaccines to prevent group B streptococcal infections. Expert Opin Biol Ther. 2003;3:975-84. http://dx.doi.org/10.1517/14712598.3.6.975

17. Bellais S, Six A, Fouet A, Longo M, Dmytruk N, Glaser P, et al. Capsular switching in group B Streptococcus $\mathrm{CC} 17$ hypervirulent clone: a future challenge for polysaccharide vaccine development. J Infect Dis. 2012;206:1745-52. http://dx.doi. org/10.1093/infdis/jis605

18. Meehan M, Cunney R, Cafferkey M. Molecular epidemiology of group B streptococci in Ireland reveals a diverse population with evidence of capsular switching. Eur J Clin Microbiol Infect Dis. 2014;33:1155-62. http://dx.doi.org/10.1007/s10096-014-2055-5

19. Farley MM. Group B streptococcal infection in older patients. Spectrum of disease and management strategies. Drugs Aging. 1995;6:293-300. http://dx.doi.org/10.2165/ 00002512-199506040-00004

20. Preventing neonatal group B streptococcal infection. Intrapartum antibiotic prophylaxis in some high-risk situations. Prescrire Intl. 2011;20:72-7.

21. Pearlman MD, Pierson CL, Faix RG. Frequent resistance of clinical group B streptococci isolates to clindamycin and erythromycin. Obstet Gynecol. 1998;92:258-61. http://dx.doi.org/10.1016/ S0029-7844(98)00155-0 
22. Martins ER, Melo-Cristino J, Ramirez M. Evidence for rare capsular switching in Streptococcus agalactiae. J Bacteriol. 2010;192:1361-9. http://dx.doi.org/10.1128/JB.01130-09

23. Huson DH, Bryant D. Application of phylogenetic networks in evolutionary studies. Mol Biol Evol. 2006;23:254-67. http://dx.doi.org/10.1093/molbev/msj030

24. Chin CS, Alexander DH, Marks P, Klammer AA, Drake J, Heiner C, et al. Nonhybrid, finished microbial genome assemblies from long-read SMRT sequencing data. Nat Methods. 2013;10:563-9. http://dx.doi.org/10.1038/nmeth.2474

25. Myers EW, Sutton GG, Delcher AL, Dew IM, Fasulo DP, Flanigan MJ, et al. A whole-genome assembly of Drosophila. Science. 2000;287:2196-204. http://dx.doi.org/10.1126/ science.287.5461.2196

26. Kent WJ. BLAT-the BLAST-like alignment tool. Genome Res. 2002;12:656-64. http://dx.doi.org/10.1101/gr.229202

27. Nusbaum C, Ohsumi TK, Gomez J, Aquadro J, Victor TC, Warren RM, et al. Sensitive, specific polymorphism discovery in bacteria using massively parallel sequencing. Nat Methods. 2009;6:67-9. http://dx.doi.org/10.1038/nmeth.1286

28. Tritt A, Eisen JA, Facciotti MT, Darling AE. An integrated pipeline for de novo assembly of microbial genomes. PLoS ONE. 2012;7:e42304. http://dx.doi.org/10.1371/journal.pone.0042304

29. Marttinen P, Hanage WP, Croucher NJ, Connor TR, Harris SR, Bentley SD, et al. Detection of recombination events in bacterial genomes from large population samples. Nucleic Acids Res. 2012;40:e6. http://dx.doi.org/10.1093/nar/gkr928

30. Alikhan NF, Petty NK, Ben Zakour NL, Beatson SA. BLAST ring image generator (BRIG): simple prokaryote genome comparisons. BMC Genomics. 2011;12:402. http://dx.doi.org/10.1186/ 1471-2164-12-402

31. Clinical and Laboratory Standards Institute. Performance standards for antimicrobial susceptibility testing. 23rd informational supplement M100-S23. Wayne (PA): The Institute; 2013.

32. Lamy MC, Zouine M, Fert J, Vergassola M, Couve E, Pellegrini E, et al. CovS/CovR of group B streptococcus: a twocomponent global regulatory system involved in virulence. Mol Microbiol. 2004;54:1250-68. http://dx.doi.org/10.1111/ j.1365-2958.2004.04365.x
33. Kreikemeyer B, Gamez G, Margarit I, Giard JC, Hammerschmidt S, Hartke A, et al. Genomic organization, structure, regulation and pathogenic role of pilus constituents in major pathogenic streptococci and enterococci. Int J Med Microbiol. 2011;301:240-51. http://dx.doi.org/10.1016/j.ijmm.2010.09.003

34. Amin A, Abdulrazzaq YM, Uduman S. Group B streptococcal serotype distribution of isolates from colonized pregnant women at the time of delivery in United Arab Emirates. J Infect. 2002;45:42-6. http://dx.doi.org/10.1053/jinf.2001.0990

35. Ekin IH, Gurturk K. Characterization of bovine and human group B streptococci isolated in Turkey. J Med Microbiol. 2006;55:517-21. http://dx.doi.org/10.1099/jmm.0.46156-0

36. Moyo SR, Maeland JA, Bergh K. Typing of human isolates of Streptococcus agalactiae (group B streptococcus, GBS) strains from Zimbabwe. J Med Microbiol. 2002;51:595-600.

37. Musser JM, Mattingly SJ, Quentin R, Goudeau A, Selander RK. Identification of a high-virulence clone of type III Streptococcus agalactiae (group B Streptococcus) causing invasive neonatal disease. Proc Natl Acad Sci U S A. 1989;86:4731-5. http://dx.doi.org/10.1073/pnas.86.12.4731

38. Brochet M, Rusniok C, Couve E, Dramsi S, Poyart C, Trieu-Cuot P, et al. Shaping a bacterial genome by large chromosomal replacements, the evolutionary history of Streptococcus agalactiae. Proc Natl Acad Sci U S A. 2008;105:15961-6. http://dx.doi.org/10.1073/ pnas.0803654105

39. Manning SD, Foxman B, Pierson CL, Tallman P, Baker CJ, Pearlman MD. Correlates of antibiotic-resistant group B streptococcus isolated from pregnant women. Obstet Gynecol. 2003;101:74-9. http://dx.doi.org/10.1016/S0029-7844(02)02452-3

40. Luan SL, Granlund M, Sellin M, Lagergard T, Spratt BG, Norgren M. Multilocus sequence typing of Swedish invasive group $\mathrm{B}$ streptococcus isolates indicates a neonatally associated genetic lineage and capsule switching. J Clin Microbiol. 2005;43:3727-33. http://dx.doi.org/10.1128/JCM.43.8.3727-3733.2005

Address for correspondence: Nahuel Fittipaldi, Public Health Ontario, Toronto Laboratories, 661 University Ave, Ste 19-099, Toronto, Ontario M5G 1M1, Canada; email: nahuel.fittipaldi@oahpp.ca

\section{Dangerous Raw Oysters}

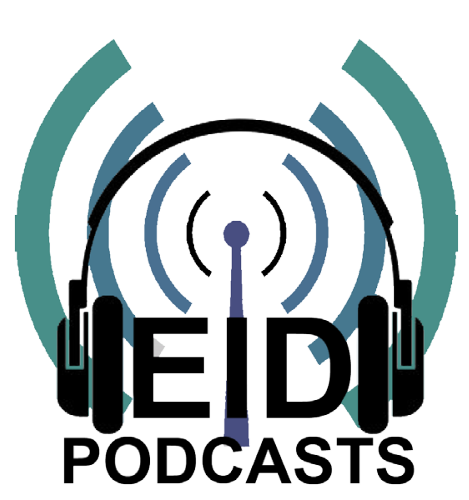
Dr. Duc Vugia, chief of the Infectious Diseases Branch at the California Department of Public Health, discusses the dangers of eating raw oysters.

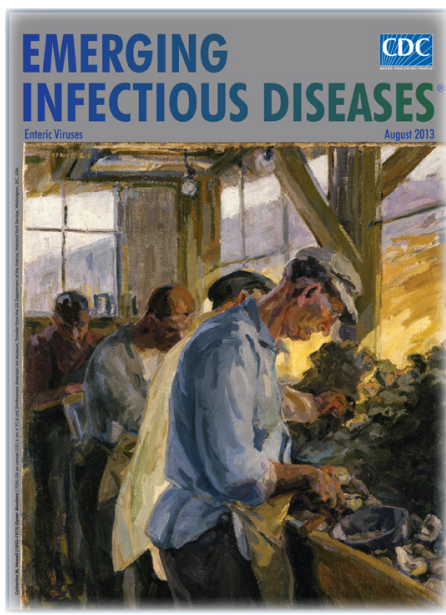

http://www2c.cdc.gov/podcasts/player.asp?f=8629455 